\title{
Borderline intellectual functioning and psychosis: Adult Psychiatric Morbidity Survey evidence
}

\author{
A. Hassiotis, M. Noor, P. Bebbington, A. Afia, J. Wieland and T. Qassem
}

\begin{abstract}
Summary
Borderline intellectual functioning (BIF) is associated with several neuropsychological deficits. We used national data to establish the prevalence of psychosis and psychotic symptoms, and the role of potential mediators. The BIF group were more than twice as likely to have probable psychosis (adjusted odds ratio $(\mathrm{OR})=2.3,95 \% \mathrm{Cl} 1.4-4.0$ ) and to report hallucinations (adjusted $\mathrm{OR}=2.9,95 \% \mathrm{Cl} 1.9-4.4$ ) but not persecutory ideation. Salient mediators were depressive symptoms and the cumulative total of life events. Our findings suggest
\end{abstract}

mechanisms other than drug use that are contributing to the strong relationship between BIF and severe mental illness and that may be amenable to treatment.

\section{Declaration of interest}

None.

\section{Copyright and usage}

(c) The Royal College of Psychiatrists 2017
Psychosis is conceptualised as a neurodevelopmental disorder, ${ }^{1}$ an argument supported by studies that have established that cognitive deficits in childhood are associated with the emergence of psychotic disorder in late adolescence or adulthood. Freeman et $a l^{2}$ argue that lower intellectual functioning may directly have an impact on the development of psychosis by altering the way in which stimuli and events are interpreted. This suggests that low IQ may be associated with specific psychotic symptoms or syndromes as in the case of persecutory ideation. Pre-existing cognitive deficits may increase vulnerability to other processes involved in the emergence of psychotic states that would meet the statistical criteria for mediation. Psychotic disorders are associated with life events (including sexual, physical and emotional abuse $^{3}$ ) and with drug use and dependence, ${ }^{4}$ and affective disturbance may increase the severity of auditory hallucinations and persecutory and grandiose delusions. ${ }^{5}$

We were interested in exploring the relationship between psychotic symptoms and the diagnostic group of borderline intellectual functioning (BIF) defined as an IQ between one and two standard deviations below the mean $(70-85) .{ }^{6} \mathrm{BIF}$ is associated with several neuropsychological difficulties distinct from those of other cognitively impaired groups. DSM- 5 has recast BIF to a V code (Axis II), denoting a condition warranting particular clinical attention. ${ }^{7}$ Despite the difficulties with ascertainment, BIF poses an important dilemma for care delivery to patients with subtle deficits. We undertook a secondary analysis of the 2000 and 2007 British national surveys of psychiatric morbidity, reported fully elsewhere, ${ }^{8,9}$ to investigate the particular association between BIF and psychosis. We predicted that individuals with BIF would: (a) have a higher prevalence of psychotic disorder than their counterparts with average intelligence; and (b) be more likely than their counterparts with average intelligence to report persecutory ideation and auditory hallucinations. If the mechanism involved in the emergence of psychotic phenomena is related to cognitive function, we expect less mediation by affective changes and by the experience of stressful antecedents.

\section{Method}

The participants were aged 16-74 years with a National Adult Reading Test $(\mathrm{NART})^{10}$ verbal IQ within the $70-85$ range. All those who had reported A-level or higher qualifications were excluded. In phase 1 of each survey, participants were screened with the Psychosis Screening Questionnaire (PSQ) ${ }^{11}$ for possible psychosis (PSQ items 3a and 5a for persecutory ideation and auditory hallucinations experienced over the past year respectively). Participants were invited to a phase 2 assessment for a definitive diagnosis of psychosis using the Schedules for Clinical Assessment in Neuropsychiatry (SCAN) system ${ }^{12}$ if they were on antipsychotic medication, were admitted to hospital in the past 3 months, had a PSQ report of auditory hallucinations or self-reported diagnosis of psychosis. Here, we present analyses based on a composite measure of 'probable psychosis' comprising individuals identified using SCAN, together with some participants from phase 1 who met at least two of the screening criteria listed above.

Possible mediators were: reported sexual abuse and bullying, a cumulative total of victimisation events and the use of cannabis, as these are repeatedly associated with psychosis. ${ }^{13}$ We also examined the role of depressive and anxiety symptoms, measured by the Revised Clinical Interview Schedule, ${ }^{14}$ which appear to mediate the relationship of traumatic causes with psychosis, in particular, sexual abuse and bullying.

Analyses were performed using Stata software Release 14. Data were weighted for survey design non-response. We used binary logistic regression to determine associations between BIF and psychosis, and the two individual psychotic symptoms. To test for our putative mediators, we used the KHB (Karlson Holm Breen ${ }^{15}$ commands in Stata, which decomposes the total effect of a variable into quantified direct and indirect effects.

\section{Results}

A total of 1701 individuals out of 15983 were identified with BIF, giving a weighted prevalence of $10.9 \%$. The weighted prevalence of probable psychosis was $0.98 \%$, compared with $0.42 \%$ for the non-BIF group (adjusted odds ratio $(\mathrm{OR})=2.3$, 95\% CI 1.4-4.0). The prevalence of auditory hallucinations in the BIF group was $2.06 \%$ compared with $0.81 \%$ in the non-BIF group (OR $=2.9$, 95\% CI 1.9-4.4). There was no difference in the prevalence of persecutory ideation in those with and without BIF $(8.9 \%$ and $8.6 \%$ respectively, OR $=1.095 \%$ CI $0.8-1.3$ ). Table 1 provides details of the odds ratios adjusted for separate confounders.

We estimated that $13.7 \%$ of the overall link between BIF and psychosis could be accounted for by the effect of BIF on hallucinations. The link between BIF and psychosis was significantly mediated by depressive but not anxiety symptoms (20.6\% v. 3.9\%). Of our three measures of stress only the cumulative measure met significance criteria for mediation, accounting for $11.2 \%$ of the 
BIF-psychosis link. The link was not mediated by a history of cannabis use. Overall, a quarter of the association between BIF and psychosis was accounted for by the effects of depression $(17.9 \%)$ and the cumulative total of life events (6\%). Similarly, depressive symptoms mediated $11.8 \%$ of the link between BIF and hallucinations. The cumulative life event score accounted for $6.7 \%$ of the link between BIF and hallucinations.

\section{Discussion}

To our knowledge, this is the first study to examine associations between a specific patient profile and psychotic symptoms. Using amalgamated data from two national psychiatric morbidity surveys, we demonstrated that BIF is significantly associated with probable psychosis and with auditory hallucinations that remained significant after controlling for age, gender, current social class and ethnicity. Counter to prediction, persecutory ideation was no more frequent in people with BIF. Given the statistical power provided by this data-set, this must be taken as a robust result. It is the most intriguing finding in our analysis, particularly as it could be argued that cognitive limitations would have an impact on social interpretation and therefore lead to mistaken perceptions of the intentions of others. There may be further scope for effects of BIF on other aspects of the psychotic syndrome including specific cognitive impairments, for which there were no measures in the national surveys.

The largest individual contribution to psychosis in the BIF group comes from the increase in non-psychotic symptoms (depressive rather than anxiety symptoms). This parallels findings from other studies of mediation effects in psychosis ${ }^{16}$ and of established associations of low IQ with depression and mixed anxiety/depression. ${ }^{17}$ Reduced IQ may be a greater risk factor for depression compared with anxiety or it may operate by altering the symptoms of mental disorder, bringing forward depressive symptoms at the expense of anxiety and may be misdiagnosed as negative symptoms. Our analyses indicate that presence of hallucinations is not a result of other diagnoses, such as borderline personality disorder or part of normal experience. ${ }^{18}$ There is no evidence that people with BIF are more prone to psychosis because of exposure to sexual abuse and bullying but a suggestion of a general excess and impact of such experiences in this group.

The national surveys provide samples large enough to allow analysis of subgroups free of the referral bias that may exist in clinical settings and use consistent methods and measures although the cross-sectional design restricts inferences about causality. The NART may lead to an overestimation of BIF or

\begin{tabular}{|c|c|c|}
\hline Confounder & OR $(95 \% \mathrm{Cl})$ & $P$ \\
\hline \multicolumn{3}{|l|}{ Psychosis } \\
\hline Unadjusted & $2.3(1.4-3.9)$ & 0.002 \\
\hline \multicolumn{3}{|c|}{ Adjusted for separate confounders } \\
\hline Age & $2.2(1.3-3.9)$ & 0.005 \\
\hline Gender & $2.3(1.3-3.9)$ & 0.004 \\
\hline Social class & $1.9(1.0-3.4)$ & 0.038 \\
\hline Ethnicity & $2.2(1.3-3.8)$ & 0.004 \\
\hline \multicolumn{3}{|l|}{ Hallucinations } \\
\hline Unadjusted & $2.9(1.9-4.4)$ & $<0.001$ \\
\hline \multicolumn{3}{|c|}{ Adjusted for separate confounders } \\
\hline Age & $2.8(1.8-4.4)$ & $<0.001$ \\
\hline Gender & $3.0(1.9-4.7)$ & $<0.001$ \\
\hline Social class & $2.6(1.7-4.2)$ & $<0.001$ \\
\hline Ethnicity & $2.9(1.9-4.4)$ & $<0.001$ \\
\hline
\end{tabular}

inclusion of people with mild intellectual disability and has not been validated in the BIF population therefore it could be a source of measure bias.

BIF is clearly associated with significant psychopathology; some factors that contribute to this psychopathology may be amenable to prompt diagnosis and treatment. Accurate characterisation of mental disorders in people with BIF is the first step towards improved care and reduction in chronic disability.

A. Hassiotis, PhD, M. Noor, MSC, P. Bebbington, PhD, A. Afia, PhD, UCL Division of Psychiatry, London, UK; J. Wieland, PhD, Poli-plus, Leiden, The Netherlands; T. Qassem, MD, Division of Mental Health and Well-being, University of Warwick, Coventry, UK

Correspondence: A. Hassiotis, UCL Division of Psychiatry, Maple House, 149 Tottenham Court Road, London W1T 7NF, UK. Email: a.hassiotis@ucl.ac.uk

First received 14 Jul 2016, final revision 7 Oct 2016, accepted 30 Jan 2017

\section{References}

1 Owen MJ, O'Donovan MC, Thapar A, Craddock N. Neurodevelopmental hypothesis of schizophrenia. Br J Psychiatry 2011; 198: 173-5.

2 Freeman D, McManus S, Brugha T, Meltzer H, Jenkins R, Bebbington P. Concomitants of paranoia in the general population. Psychol Med 2011; 41: 923-36.

3 Beards S, Gayer-Anderson C, Borges S, Dewey ME, Fisher HL, Morgan C. Life events and psychosis: a review and meta-analysis. Schizophr Bull 2013; 39: 740-7.

4 Hassiotis A, Ukoumunne O, Tyrer P, Piachaud J, Gilvarry C, Harvey K, et al Prevalence and characteristics of patients with severe mental illness and borderline intellectual functioning. Report from the UK700 randomised controlled trial of case management. Br J Psychiatry 1999; 175: 135-40.

5 Smith B, Fowler DG, Freeman D, Bebbington P, Bashforth H, Garety P, et al. Emotion and psychosis: links between depression, self-esteem, negative schematic beliefs and delusions and hallucinations. Schizophr Res 2006; 86: 181-8.

6 American Psychiatric Association. Diagnostic and Statistical Manual of Mental Disorders (4th edn) (DSM-IV). APA, 1994.

7 Wieland J, Zitman FG. It is time to bring borderline intellectual functioning back into the main fold of classification systems. BJPsych Bull 2016; 40: 204-6.

8 Singleton N, Bumpstead R, O'Brien M, Lee A, Meltzer H. Psychiatric morbidity among adults living in private households, 2000. Int Rev Psychiatry 2003; 15: 65-73.

9 McManus S, Meltzer H, Brugha T, Bebbington $\mathrm{P}$, Jenkins R. Adult Psychiatric Morbidity in England, 2007: Results of a Household Survey. The Health \& Social Care Information Centre, Social Care Statistics, 2009.

10 Berry DT, Carpenter GS, Campbell DA, Schmitt FA, Helton K, Lipke-Molby T. The New Adult Reading Test-Revised: accuracy in estimating WAIS-R IQ scores obtained 3.5 years earlier from normal older persons. Arch Clin Neuropsychology 1994; 9: 239-50.

11 Bebbington PE, Nayani T. The Psychosis Screening Questionnaire. Int J Meth Psychiatr Res 1995; 5: 11-20.

12 World Health Organization. SCAN: Schedules for Clinical Assessment in Neuropsychiatry. WHO, 1992.

13 Gracie A, Freeman D, Green S, Garety PA, Kuipers E, Hardy A, et al The association between traumatic experience, paranoia and hallucinations: a test of psychological models. Acta Psychiatr Scand 2007; 116: 280-9.

14 Lewis G, Pelosi AJ, Araya R, Dunn G. Measuring psychiatric disorder in the community: a standardised assessment for use by lay interviewers. Psychol Med 1992; 22: 465-86.

15 Karlson KB, Holm A, Breen R. Comparing regression coefficients between same-sample nested models using logit and probit. A new method. Sociol Methodol 2011; 42: 286-313.

16 Marwaha S, Broome MR, Bebbington PE, Kuipers E, Freeman D. Mood instability and psychosis: analyses of British national survey data. Schizophr Bull 2014; 40: 269-77.

17 Rai D, Stansfeld S, weich S, Stewart R, McBride O, Brugha T, et al. Chapter 13: Comorbidity in mental and physical illness. In Adult Psychiatric Morbidity Survey: Survey of Mental Health and Wellbeing 2014 (eds S McManus, P Bebbington, R Jenkins, T Brugha): 323-47. Health and Social Care Information Centre, 2016.

18 Upthegrove R, Broome MR, Caldwell K, Ives J, Oyebode F, Wood SJ. Understanding auditory verbal hallucinations: a systematic review of current evidence. Acta Psychiatr Scand 2016; 133: 352-67. 